\title{
Nota complementar sobre a composição ictiofaunística da Baía de Guaratuba, Paraná, Brasil ${ }^{1}$
}

\author{
Paulo T.C. Chaves ${ }^{2}$ \\ Ana Lúcia Vendel ${ }^{3}$
}

\begin{abstract}
A complementary note about the icthyofaunistic composition of the Guaratuba Bay, Paraná, Brazil. The utilisation of multiple gears and the surveying of other areas than that of mangrove habitats have increased in 27 the number of fish species known in the Guaratuba Bay, an estuarine ecosystem located in the southern of Brazilian coastal region (25'52'S, $48^{\circ} 39^{\prime} \mathrm{W}$ ). The new occurrence of a typically freshwater species (Pimelodidae) and of several Clupeiformes and Gobiidae species, reveals the importance of the salt marsh and the innermost zones of this Bay to the distribution of certain fish groups.

KEY WORDS. Fish, estuaries, Guaratuba Bay
\end{abstract}

CHAves \& CORRÊA (1998) fornecem uma lista de espécies de peixes ocorrentes na Baía de Guaratuba, Estado do Paraná (2552'S, 48³9'W), baseados em coletas realizadas entre 1993 e 1996 com redes tipo tarrafa, emalhe e arrasto de fundo com portas. Uma espécie de raia e 59 de teleósteos integram o inventário, o primeiro já divulgado sobre a região. Mais recentemente (janeiro e fevereiro de 1997, e abril de 1998 a fevereiro de 2000), novas operações de pesca experimental foram realizadas na Baía. Elas abrangeram uma área maior que a primeira, estendendo-se da extremidade oeste da Baía (foz do Rio Guanxuma, um braço do Rio Cubatão) à Ilha da Sepultura, próximo à conexão com o mar. Um mapa da região está disponível em CHAVES \& VENDEL (1997), CHAVES \& CORRÊA $(1998,2000)$, por exemplo. Realizaram-se amostragens com redes: arrasto manual de praia, tipo picaré ( $30 \mathrm{~m}$ de comprimento, $3 \mathrm{~m}$ de altura e malha $5 \mathrm{~mm}$ entre nós opostos), abrangendo áreas rasas de marismas; de arrasto com portas (malha $20 \mathrm{~mm}$ no ensacador) e de emalhe (malha $70 \mathrm{~mm}$ ), estas últimas em setores com profundidade até $6 \mathrm{~m}$. Tal esforço permitiu aumentar em $45 \%$ o número de espécies conhecidas na Baía de Guaratuba, o qual passou de 60 para 87. A identificação baseou-se em Figueiredo \& MENEZES (1978; 1980; 2000), MENEZES \& Figueiredo (1980, 1985) e CERVIGÓN et al. (1992), e a classificação taxonômica seguiu NELSON (1994). A maioria dos exemplares foi preservada e encontra-se depositada na coleção ictiológica do Museu de História Natural Capão da Imbuia (MHNCI), pertencente à Prefeitura Municipal de Curitiba. Exemplares de certas espécies, inicialmente não reconhecidos como sendo novas ocorrências para a região, foram descartados antes que pudessem ser preservados para depósito em coleção.

1) Contribuição número 1297 do Departamento de Zoologia, Universidade Federal do Paraná.

2) Departamento de Zoologia, Universidade Federal do Paraná. Caixa Postal 19020, 81531-990 Curitiba, Paraná, Brasil. E-mail: poissons@cce.ufpr.br. Bolsista do CNPq

3) Curso de Pós-Graduação em Zoologia, Universidade Federal do Paraná. Bolsista do CNPq. E-mail: analucia@cem.ufpr.br 
A relação seguinte apresenta as espécies cujo registro é inédito para a Baía de Guaratuba. Assinalam-se com "M" as espécies coletadas em áreas marginais (picaré) e com "NM" as coletadas em áreas não marginais (arrasto de portas e emalhe):

\section{Clupeiformes}

Clupeidae

Brevoortia pectinata (Jenyns, 1842). MHNCI 8998. Savelha. (NM)

Sardinella brasiliensis (Steindachner, 1789). Não depositado. Sardinha. (M)

Engraulididae (= Engraulidae)

Anchoa parva (Fowler, 1976). MHNCI 8999. Manjuba. (M)

Anchoa lyolepis (Evermann \& Marsh, 1902). MHNCI 8999. Manjuba. (M)

Anchoviella lepidentostole (Fowler, 1911). MHNCI 9000. Manjuba. (M)

\section{Siluriformes}

Pimelodidae

Rhamdia quelen (Quoy \& Gaimard, 1824). MHNCI 8998. Jundiá. (NM)

\section{Gasterosteiformes}

Syngnathidae

Syngnathus rousseau Kaup, 1856. MHNCI 8999. Peixe-cachimbo. (M)

\section{Gadiformes}

Gadidae

Urophycis brasiliensis (Kaup, 1858). MHNCI 8998. Abrótea. (NM)

\section{Atheriniformes}

Atherinopsidae

Atherinella brasiliensis (Quoy \& Gaimard, 1824). MHNCI 8999. Peixe-rei. (M)

\section{Beloniformes}

Belonidae

Strongylura timucu (Walbaum, 1792). MHNCI 8999. Agulha. (M)

S. marina (Walbaum, 1792). MHNCI 9000. Agulha. (M)

Hemirhamphidae

Hyporhamphus unifasciatus (Ranzani, 1842). MHNCI 9000. Agulha. (M)

\section{Perciformes}

Gerreidae

Ulaema lefroyi (Goode, 1874). Não depositado. Carapicu. (M)

Carangidae

Oligoplites palometa (Cuvier, 1833). Não depositado. Salteira, guaivira. (M)

O. saliens (Bloch, 1793). Não depositado. Salteira, guaivira. (M)

O. saurus (Bloch \& Schneider, 1801). MHNCI 9000. Salteira, guaivira. (M)

Selene setapinnis (Mitchill, 1815). MHNCI 8998. Peixe-galo. (NM) 
Gobiidae

Gobioides braussonnetii Lacépède, 1800. MHNCI 8999. (M)

Gobionellus oceanicus (Pallas, 1770). Não depositado. Amborê. (M)

G. shufeldti (Jordan \& Eigenmann, 1886). MHNCI 8999. (M)

G. smaragdus (Valenciennes, 1837). MHNCI 9000. (M)

G. stigmaticus (Poey, 1861). Não depositado. (M)

G. stomatus (Starrks, 1913). Não depositado. (M)

Microgobius meeki Evermann \& Marsh, 1900. MHNCI 9000. (M)

Scombridae

Scomberomorus brasiliensis Collette, Russo \& Zavala-Camin, 1978. MHNCI 8999. Sororoca. (M)

\section{Tetraodontiformes}

Diodontidae

Cyclichthys spinosus (Linnaeus, 1758). Não depositado. Baiacu-de-espinho. (M)

Tetraodontidae

Sphoeroides greeleyi Gilbert, 1900. MHNCI 8999. Baiacu. (M)

\section{Considerações finais}

A exploração de uma área maior e de hábitats diferentes dos pesquisados no trabalho de CHAVES \& CORRÊA (1998), assim como a adição de outro tipo de aparelho de captura, o arrasto manual, explicam os novos registros aqui apresentados. A captura com rede de praia em área de marismas, por exemplo, relaciona-se em particular aos engraulidídeos, aterinídeos e gobiídeos, abundantes naqueles biótopos. Nas operações na foz do Rio Guanxuma, num verão particularmente chuvoso, foi coletada uma espécie dulceaqüíola (Rhamdia quelen), até então não registrada na Baía. Considerando o tempo total já investido, bem como a extensão da área pesquisada e a variedade de aparelhos empregados, conclui-se que a composição ictiofaunística permanente da Baía de Guaratuba esteja hoje bem conhecida. Entretanto, é possível que espécies marinhas de ocorrência esporádica ainda não tenham sido registradas, visto seu ingresso em caráter apenas ocasional, difícil de ser assinalado em programas de captura experimental.

AGRADECIMENTOS. Aos pescadores envolvidos no trabalho de campo, João Carlos da Costa e Itamar da Costa; ao MSc. Vinícius Abilhôa, curador da coleção ictiológica do Museu de História Natural Capão da Imbuia; à FUNPAR/UFPR, CAPES e CNPq por auxílios concedidos.

\section{REFERÊNCIAS BIBLIOGRÁFICAS}

Cervigón, F.; R. Cipriani; W. Fischer; L. Garibaldi; M. Hendrickx; A.J. Lemus; R. Márquez; J.M. Poutiers; G. Robaina \& B. Rodrigulz. 1992. Guía de Campo de las Especies Comerciales Marinas y de Aguas Salobres de la Costa Septentrional de Sur America. Roma, FAO, 513p.

Chaves, P.T.C. \& C.E. Correa. 2000. Temporary use of a coastal ecosystem by the fish, Pomadasys corvinaeformis (Perciformes: Haemulidae) at Guaratuba Bay, Brazil. Rev. Brasil. Oceanogr. 48 (1): $1-7$. 
Chaves, P.T.C. \& M.F.M. CorrêA. 1998. Composição ictiofaunística da área de manguezal da Baía de Guaratuba, Paraná, Brasil. Revta bras. Zool. 15 (1): 195-202.

Chaves, P.T.C. \& A.L. Vendel. 1997. Indicadores reprodutivos das espécies de Citharichthys Bleeker

(Teleostei, Pleuronectiformes) na Baía de Guaratuba, Paraná, Brasil. Revta bras. Zool. 14 (1): 73-79.

Figueiredo, J.L. \& N.A. Menezes. 1978. Manual de Peixes Marinhos do Sudeste do Brasil. II.

Teleostei (1). São Paulo, Museu de Zoologia, Univ. São Paulo, 110p. 1980. Manual de Peixes Marinhos do Sudeste do Brasil. II. Teleostei (2). São Paulo, Museu de Zoologia, Univ. São Paulo, 90p.

- 2000. Manual de Peixes Marinhos do Sudeste do Brasil. VI. Teleostei (5). São Paulo, Museu de Zoologia, Univ. São Paulo, 116p.

Menezes, N.A. \& J.L. Figueiredo. 1980. Manual de Peixes Marinhos do Sudeste do Brasil. IV. Teleostei (3). São Paulo, Museu de Zoologia, Univ. São Paulo, 96p.

. 1985. Manual de Peixes Marinhos do Sudeste do Brasil. IV. Teleostei (4). São Paulo, Museu de Zoologia, Univ. São Paulo, 105p.

Nel.Son, J.S. 1994. Fishes of the World. New York, John Wiley \& Sons Inc., XX+600p.

Recebido em 28.XII.2000; aceito em 26.VI.2001. 